\title{
FIB sample preparation of thin films and soft materials
}

\author{
A.M. Minor* \\ *Department of Materials Science and Engineering, University of California, Berkeley \\ and National Center for Electron Microscopy, Lawrence Berkeley National Laboratory, \\ One Cyclotron Road, MS 72, Berkeley, CA 94720
}

Focused ion beam (FIB) instrumentation has proven to be extremely useful for preparing cross-sectional samples for transmission electron microscopy (TEM) investigations. The two most widely used methods involve either milling a trench into a thin film/substrate sample while leaving behind an electron-transparent window, or the so-called "lift-out" method. While these two methods are very powerful in their versatility and ability to make site-specific TEM samples, they also rely on using a sacrificial layer to protect the surface of the sample as well as the removal of a relatively large amount of material, depending on the size of the initial sample. While neither of these two characteristics are typically seen as drawbacks, some samples prohibit the use of a sacrificial layer or long milling times. Therefore, this tutorial will also introduce a relatively new strategy for making thin film cross-sections with the FIB, known as Shadow FIBing [1]. In this technique, the corner of a sample is positioned at an angle of incidence not normal to the electron beam. As seen in Figure 1, the thin film is positioned so that it is in the "shadow" of the ion beam. This tutorial will discuss these main strategies for FIB preparation of cross-sectional thin film samples and discuss the advantages and disadvantages of each method.

In addition to covering the main issues related to FIB milling of traditional hard materials (metals and ceramics), particular attention will be paid to the FIB preparation of polymer thin film samples. Understanding the microstructure of polymer thin films on hard substrates is important for many organic electronic applications, and since polymers are easily damaged by the incident ion beam strategies for limiting FIB damage of the polymer thin films is particularly important. One of the main advantages of FIB preparation for thin film analysis is the ability to readily cross-section dissimilar materials. Traditional TEM sample preparation techniques such as microtomy or broadbeam ion milling are not ideal for cross-sectioning all of the device components in a manner that retains the structure of all components equally. Organic electronic thin film systems are particularly difficult to section with other sample preparation methods since they often contain ceramic, polymer and metallic thin films stacked together, as shown in Figure 2. Characterization of these complex systems requires investigation of the internal polymer structure as well as the interface between the polymer this films and the substrate [2]. This tutorial will present experiments in which a focused ion beam (FIB) was used to prepare cross-sectional TEM samples of polymer thin films in order to systematically investigate the optimal parameters for successful TEM sample preparation in these hybrid systems. Understanding and limiting FIB damage of the polymer thin films is particularly important, since polymers are easily damaged by the high energy Ga ion beam $(30 \mathrm{keV})$. In addition to direct damage from the incident beam, the low thermal 
conductivity of polymers as compared to metals and ceramics can lead to significant beam heating.

\section{References}

[1] S. Welz, et. al, Microsc. Microanal., 11, 834 (2005)

[2] M.J. Park, et. al, Advanced Materials, 21, 203 (2009)

[3] This research was supported by the Scientific User Facilities Division of the Office of Basic Energy Sciences, U.S. Department of Energy under Contract \# DE-AC0205CH11231.
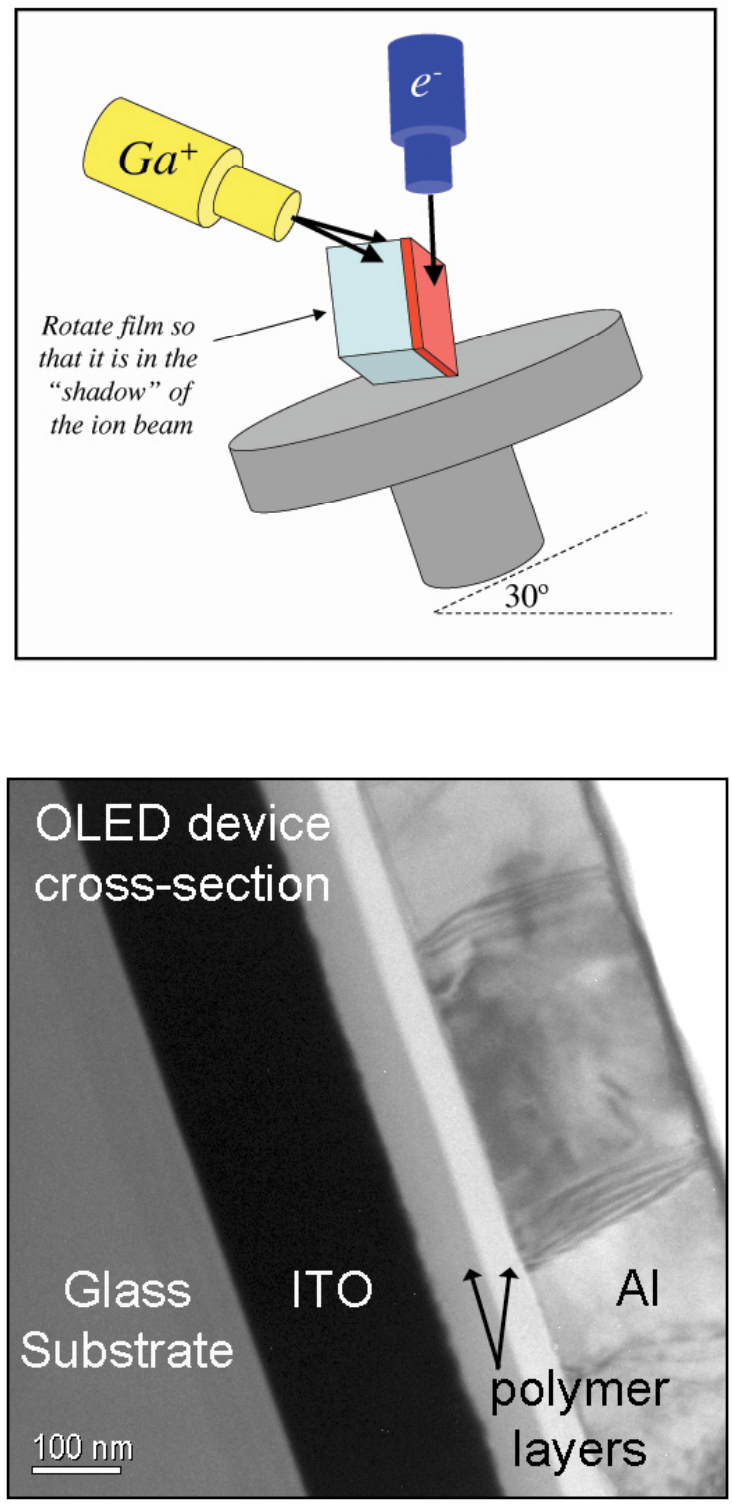

FIG. 1. Schematic of the Shadow-FIB technique for cross-sectional TEM sample preparation. In this case, a thin film (red) is tilted so that the surface is in the "shadow" of the ion beam. This geometry allows for the ability to cross-section a sample without using a sacrificial layer or long milling times. The ion beam is focused on either side of a corner of the sample, resulting in an electron-transparent wedge ending on the film surface. The angle of incidence of the ion beam can be varied depending on the circumstances, as long as it is directed from the back, substrate-side.

FIG. 2. TEM image of an Organic Light Emitting Diode (OLED) device crosssectioned with the FIB. In this case, the shadow FIB method was used to section through the multiple dissimilar materials without the use of a sacrificial layer. 